voluntary resettlement is sanctioned within demarcated Tiger Reserves in India, actual implementation is often delayed for various reasons. The Wildlife Conservation Society (WCS) India Program and its partners, with help from WCS, New York, have participated in encouraging the implementation of relocation activities in Dandeli-Anshi Tiger Reserve, in the state of Karnataka, south-west India. Since its demarcation as a Tiger Reserve in $2007,>600$ families resident within the reserve have expressed interest in resettlement. However, various hurdles have prevented the Government of Karnataka from facilitating this.

WCS India has catalysed and provided strategic support for the implementation of the relocation programme, focusing on a remote settlement, Naiphed, within the Reserve. These efforts culminated in May 2014, with the village now relocated outside the protected area. The relocation followed models developed through previous involvement of WCS India in the resettlement of c. 1,000 families elsewhere in the Malenad-Mysore landscape, which spans the states of Karnataka, Kerala and Tamil Nadu. Naiphed comprised three families and was formerly located c. $10 \mathrm{~km}$ from amenities such as public transport, medical facilities and schools, and was without electricity. The village suffered repeated incidents of crop raiding by recovering wildlife populations, and the infertile land produced only low yields of rice and Areca catechu. The resettled families now have access to fertile agricultural land and amenities. Following the norms laid down by the Government of Karnataka, WCS provided each family with appropriate financial compensation to facilitate adaptation to their new home.

As a result of the resettlement all land formerly occupied by the village has been relinquished to the Government of Karnataka. Regeneration of native vegetation will follow, turning the abandoned village into habitat for tigers, their prey and other fauna. The Malenad-Mysore Tiger Landscape, which comprises multiple protected areas, is one of the most promising conservation landscapes for the long-term conservation of the tiger. Included in this landscape is the Nagarahole-Bandipur complex of contiguous forests, which has a tiger density of $10-15$ individuals per $100 \mathrm{~km}^{2}$.

Following the successful resettlement of the families from Naiphed, the government has initiated resettlement of an additional 30 families from within the Reserve. Preliminary procedures have been initiated, with some financial compensation provided to the families while subsequent steps are undertaken for fair resettlement. It is expected that the remaining families that have expressed interest in being resettled will soon follow. Resettlement programmes, when conducted in a manner that benefits villagers otherwise separated from essential services, can serve as a valuable conservation tool to hasten recovery of threatened species, alleviate threats to biodiversity and foster a positive attitude towards wildlife conservation through human-wildlife conflict mitigation.

P.M. MuthanNa and DIVYA VASUdEV Wildlife Conservation
Society, India Program, Bangalore, India
E-mail vasudev.divya@gmail.com

M.C. Vinay Kumar Centre for Wildife Studies, Bangalore, India

\section{Conservation and reintroduction of Firmiana danxiaensis, a rare tree species endemic to southern China}

Firmiana danxiaensis $\mathrm{H}$. H. Hsue \& H. S. Kiu, a tree species belonging to the Family Sterculiaceae, was discovered and named in 1987. It was listed as a Second Class Protected Key Wild Plant of China in 1999 and in the Conservation Programme for Wild Plants with Extremely Small Populations in China in 2012. It occurs only on Danxia Mountain, a natural World Heritage site in Renhua County, Guangdong Province, southern China, in an area of $168 \mathrm{~km}^{2}$. In $1987,<100$ wild individuals were known. Our investigations in 2014, however, have indicated that the population is c. 10,000 wild individuals. However, the species is threatened as it is a valuable economic species (it is an excellent garden ornamental tree and its trunk is suitable for use in piano construction).

This rare species has low genetic diversity and low population differentiation as a result of its restricted range and the strong selective pressure exerted by the soils of Danxia Mountain, which have a low soil fertility and strong acidity. In October 2011 we collected seeds from the natural population and propagated them at the nursery in Danxia Park. About 300 seedlings had grown to $\mathrm{c} .73 \mathrm{~cm}$ tall after 18 months. In May 2014, to examine the feasibility of augmenting the natural population of this tree, 45 seedlings were transplanted to the original collection site. Thirty seedlings were also transplanted to the South China Botanical Garden in Guangzhou City (c. $260 \mathrm{~km}$ south) and Tianxin Natural Reserve (c. $200 \mathrm{~km}$ west) in Lianzhou City. To date, the transplanted seedlings have grown well. Our studies on artificial propagation, reintroduction, and the ecophysiology of this tree will provide a basis for the design of an integrated species conservation plan, including in situ and ex situ conservation, and reintroduction.

QIANMEI Zhang South China Botanical Garden, Chinese Academy of Sciences. Guangzhou, China

E-mailzqm@scib.ac.cn

XIAOYING LUO School of Tourism and Geography, Shaoguan University, Shaoguan, China

ZAIXIONG CHEN Shaoguan Danxiashan Tourism Investment \& Management Co. Ltd, Shaoguan, China 